\title{
Evaluation and Optimization of Electron Capture Dissociation Efficiency in Fourier Transform Ion Cyclotron Resonance Mass Spectrometry
}

\author{
Melinda A. McFarland,* Michael J. Chalmers, John P. Quinn, \\ Christopher L. Hendrickson, ${ }^{*}$ and Alan G. Marshall* \\ Ion Cyclotron Resonance Program, National High Magnetic Field Laboratory, Florida State University, \\ Tallahassee, Florida, USA
}

\begin{abstract}
Electron capture dissociation (ECD) efficiency has typically been lower than for other dissociation techniques. Here we characterize experimental factors that limit ECD and seek to improve its efficiency. Efficiency of precursor to product ion conversion was measured for a range of peptide ( $\sim 15 \%$ efficiency) and protein ( $\sim 33 \%$ efficiency) ions of differing sizes and charge states. Conversion of precursor ions to products depends on electron irradiation period and maximizes at $\sim 5-30 \mathrm{~ms}$. The optimal irradiation period scales inversely with charge state. We demonstrate that reflection of electrons through the ICR cell is more efficient and robust than a single pass, because electrons can cool to the optimal energy for capture, which allows for a wide range of initial electron energy. Further, efficient ECD with reflected electrons requires only a short $(\sim 500 \mu \mathrm{s})$ irradiation period followed by an appropriate delay for cooling and interaction. Reflection of the electron beam results in electrons trapped in or near the ICR cell and thus requires a brief $(\sim 50 \mu \mathrm{s})$ purge for successful mass spectral acquisition. Further electron irradiation of refractory precursor ions did not result in further dissociation. Possibly the ion cloud and electron beam are misaligned radially, or the electron beam diameter may be smaller than that of the ion cloud such that remaining precursor ions do not overlap with the electron beam. Several ion manipulation techniques and use of a large, movable dispenser cathode reduce the possibility that misalignment of the ion and electron beams limits ECD efficiency. (J Am Soc Mass Spectrom 2005, 16, 1060-1066) (C) 2005 American Society for Mass Spectrometry
\end{abstract}

$\mathrm{E}$ lectron capture dissociation (ECD) $[1,2]$ is a relatively new MS/MS technique that has become popular because it provides better peptide and protein sequence coverage compared with other dissociation methods, and retains labile post-translational modifications (e.g., glycosylation $[3,4]$ and phosphorylation [5-8]. Recent applications also include fatty acids [9], antibiotic/protein complexes [10], ubiquitination [11], oligonucleotides [12], and histones [13-15]. Despite its proven analytical utility, ECD suffers from limited conversion efficiency of precursor to product ions. The dispenser cathode electron source has decreased the irradiation period and increased the repro-

Published online May 23, 2005

Address reprint requests to Dr. A. G. Marshall, Ion Cyclotron Resonance Program, National High Magnetic Field Laboratory, Florida State University, 1800 E. Paul Dirac Drive, Tallahassee, FL 32310-4005, USA. E-mail: marshall@magnet.fsu.edu

* Also members of the Department of Chemistry and Biochemistry, Florida State University, Tallahassee, FL, USA. ducibility of ECD [16], but has not significantly improved the efficiency [17]. Factors such as charge neutralization and larger number of observed fragmentation pathways make ECD difficult to apply to large precursor ions of low abundance. In addition, it can be quite difficult to establish and maintain optimized ECD operating conditions. The emergence of FT-ICR instruments in biological mass spectrometry makes the robustness and efficiency of ECD even more critical.

In this work, we systematically characterize experimental factors that limit ECD and seek to improve its efficiency. Research is designed to investigate all aspects of the ECD experiment, including duration of electron irradiation, electron energy and flux, multiple reflection of the electron beam versus a single pass through the ICR cell, ejection of trapped electrons from the ICR cell following the ECD event, and spatial overlap of the electron beam with the trapped ions. We report our conditions for optimized ECD and discuss their physical rationale and general implications. 


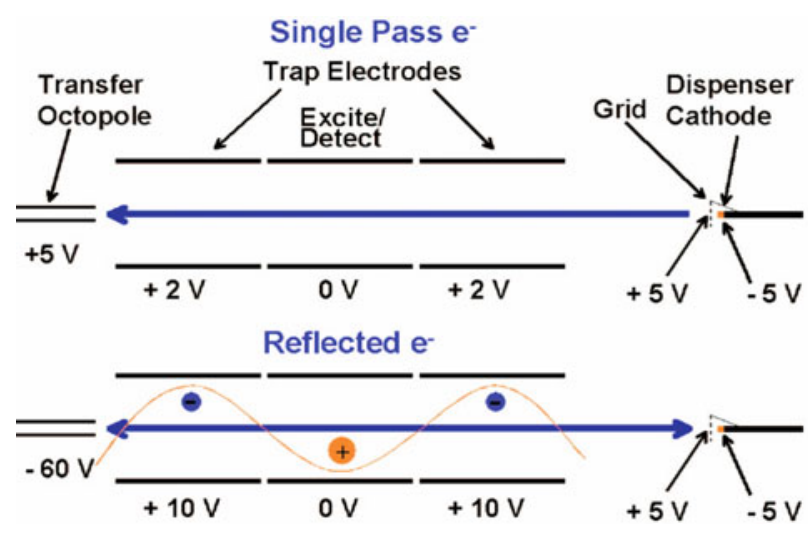

Figure 1. Schematic diagram of the potential biases for: (top) single pass ECD in which electrons make one trip through the ICR cell (the DC offset of the transfer octopole is positively biased for single pass ECD such that electrons that have passed through the cell are deposited on the octopole rods); (bottom) reflected electrons, in which electrons are reflected between the negatively biased transfer octopole and the dispenser cathode. Excitation and detection parameters are the same for both.

\section{Methods}

\section{Sample Preparation}

Substance P, glu-fibrinopeptide, melittin, bovine ubiquitin, formic acid, and acetic acid were purchased from Sigma (St. Louis, MO) and used without further purification. Methanol, acetonitrile, and water (HPLC grade) were purchased from J. T. Baker (Philipsburg, NJ). For electrospray, aqueous stock solutions were diluted to a concentration of $1 \mu \mathrm{M}$ in either 1:1 methanol:water with $2.5 \%$ acetic acid, or 1:1 acetonitrile:water with $0.1 \%$ formic acid.

\section{Fourier Transform Ion Cyclotron Resonance Mass Spectrometry}

Samples were infused into a passively shielded 9.4 tesla (220 mm bore diameter) ESI-Q-FT-ICR mass spectrometer [18] at a flow rate of $300-350 \mathrm{~nL} / \mathrm{min}$ through a $50-\mu \mathrm{m}$ i.d. fused silica capillary with a tip mechanically ground to a uniformly thin wall [19]. For external ion accumulation [20-22], precursor ions were held in a focusing octopole $\left(4.8 \mathrm{~mm}\right.$ i.d., $20 \mathrm{~cm}$ long, $300 \mathrm{~V}_{p-p}$ at $1.5 \mathrm{MHz}$ ) and transferred through an external quadrupole mass filter for precursor ion mass selection, then deposited into an accumulation octopole $(4.8 \mathrm{~mm}$ i.d., $15 \mathrm{~cm}$ long, $300 \mathrm{~V}_{p-p}$ at $1.5 \mathrm{MHz}$ ) modified for improved axial ejection [23]. The accumulation period ranged from 2 to $5 \mathrm{~s}$. Ions were then transferred to an openended cylindrical Penning trap (94 mm i.d., $300 \mathrm{~mm}$ long) [24] via an octopole ion guide $(4.8 \mathrm{~mm}$ i.d., $160 \mathrm{~cm}$ long, $125 \mathrm{~V}_{p-p}$ at $1.5 \mathrm{MHz}$ ) for electron capture dissociation. For experiments employing sequential ECD events (Figure 6) residual precursor ions in the ICR cell were isolated by stored waveform inverse Fourier transform (SWIFT) excitation [25, 26]. Product ions were subjected to frequency-sweep excitation (typically
$72 \mathrm{kHz}$ to $720 \mathrm{kHz}$ at $150 \mathrm{~Hz} / \mu \mathrm{s}$ ) and broadband detection (818 ms detection period, $512 \mathrm{~K}$ word data points). The digitized time-domain transient signal was baseline corrected, Hanning apodized, zero-filled, and Fourier transformed to yield a magnitude-mode frequency spectrum that was converted to a mass-tocharge ratio spectrum by the quadrupolar approximation [27, 28]. The experimental event sequence was controlled by a modular ICR data acquisition and analysis system (MIDAS) [29].

\section{Electron Capture Dissociation}

Electron irradiation was performed with a $1 \mathrm{~cm}$ diameter dispenser cathode [16] and molybdenum grid (80\% transmission, $8 \mathrm{~mm}$ in front of the cathode) (Heat Wave, Watsonville, CA) mounted $73 \mathrm{~cm}$ from the ICR cell center (positioned for concurrent access of an off-axis IR-laser) [17]. In some experiments, a probe-mounted dispenser cathode allowed independent adjustment of the cathode axial (from 28 to $88 \mathrm{~cm}$ from cell center) and radial ( 0 to $2.5 \mathrm{~cm}$ ) displacement from field center (the probe is for diagnostic purposes only, because the arrangement does not allow IRMPD to be performed). During all reflected electron ECD events, the trap electrodes were biased at $+10 \mathrm{~V}$, the dispenser cathode grid at $+5 \mathrm{~V}$, and the cathode ranged from $-2 \mathrm{~V}$ for higher charge states to $-5 \mathrm{~V}$ for doubly charged precusor ions. The transfer octopole was typically set to -60 $\mathrm{V}$ throughout the experiment, causing all electrons that passed through the ICR cell to be reflected back toward the dispenser cathode. In one set of experiments, the transfer octopole bias was $+5 \mathrm{~V}$ during the ECD event, so that electrons were collected on the octopole rods after one pass through the ICR cell (see Figure 1). The duration of electron irradiation was optimized for each analyte (Figure 2, Substance P) and was typically $30 \mathrm{~ms}$ for doubly protonated Substance P, $15 \mathrm{~ms}$ for quadru-

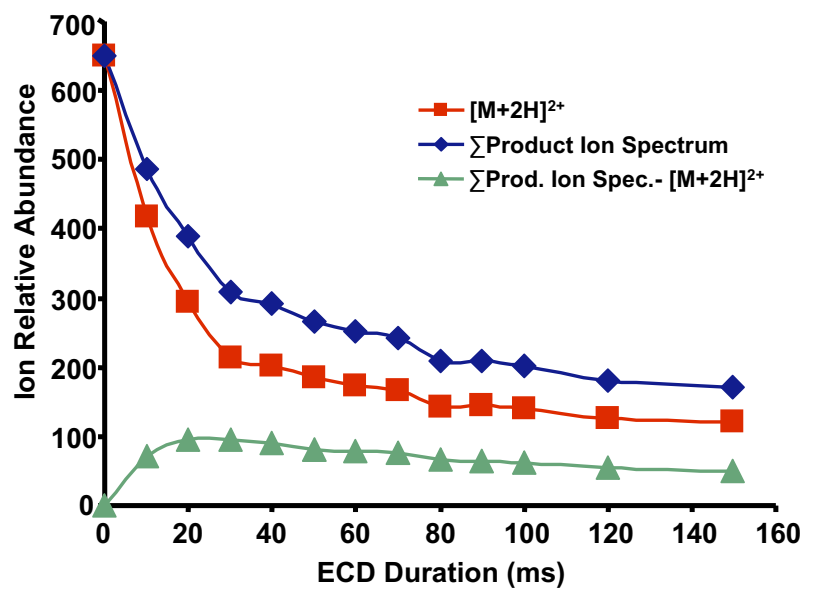

Figure 2. Abundances of precursor ion (filled square), total ions (filled diamond), and fragment ions (filled triangle) in the product ion spectrum versus electron irradiation period for reflected electrons (see text) . 
ply protonated melittin, and $5 \mathrm{~ms}$ for all charge states of ubiquitin (i.e., the optimum irradiation period varies inversely with charge state). The electron energy was varied by changing the cathode voltage while keeping the grid voltage $(+5 \mathrm{~V})$ and ICR cell voltages $(+10 \mathrm{~V})$ constant.

Electron irradiation was followed by removal of any remaining electrons from the ICR cell, by either of two equally effective methods: in the first, the trap plates were lowered to $+2 \mathrm{~V}$, the grid remained at $+5 \mathrm{~V}$, and the cathode was biased to $+10 \mathrm{~V}$ (typically for $1 \mathrm{~ms}$ ); in the second, the trap plates were pulsed to a more negative potential (typically 0 to $-10 \mathrm{~V}$ ) for $50 \mu \mathrm{s}$ (similar to suspended trapping [30]. At all other times, the cathode bias voltage was $-0.1 \mathrm{~V}$ and the grid potential was $-200 \mathrm{~V}$, to ensure that unwanted electrons did not leak from the cathode into the ICR cell. For excitation and detection, trap electrodes were biased at $+2 \mathrm{~V}$ for all experiments.

Fragmentation efficiency was calculated as the sum of all electron capture product abundances divided by the initial precursor ion abundance (measured in a separate experiment):

$$
\begin{aligned}
\mathrm{E}_{\text {Frag }}= & {\left[\sum\left(\text { Productionspectrum }-[\mathrm{M}+\mathrm{nH}]^{\mathrm{n}+}\right) /\right.} \\
& \left.\left.\sum \text { (Precursorionspectrum }\right)\right] * 100
\end{aligned}
$$

Further, note that ECD reduces the parent ion charge by the factor, $(n-1) / n$, in which $n$ is the number of charges on the precursor ion, thereby reducing the total signal available in an FT-ICR product ion spectrum. The electron capture efficiency is therefore related to the measured ion relative abundances (see Figure 3)

$$
\mathrm{E}_{\text {Capt }}=\mathrm{E}_{\text {frag }}(\mathrm{n} /(\mathrm{n}-1))
$$

Proper comparison of ECD efficiencies in the literature is difficult, not only because the hardware and methodology differ, but also because efficiency has been calculated differently. We propose that the present definitions are the most logical and useful parameters by which ECD efficiency can be measured.

\section{Results and Discussion}

\section{Inherent Limitations}

Perhaps the most compelling attribute of ECD is that it does not show a preference for labile bonds and therefore tends to cleave the peptide backbone randomly and retain post-translational modifications. However, increase in the number of observed fragments means that the available precursor ion signal must be distributed over more dissociation channels so that the abundance of each fragment type is smaller. In addition, unlike other dissociation methods, capture of an electron necessarily results in charge neutralization and, therefore, further reduction of the available precursor ion signal. Charge neutralization is most limiting for doubly- charged precursor ions, for which electron capture reduces the observable fragment ion signal by $50 \%$. That reduction is particularly detrimental for trace analysis (for which the precursor ion signal is low) and for LC MS/MS (for which signal averaging is difficult). The combined impact of random cleavage with charge neutralization makes it particularly important to maximize the conversion of precursor ion to product ion.

\section{Efficiency}

Typical ECD efficiency for peptides of various size is shown in Figure 3. Efficiency generally increases with charge state, attributable (at least in part) to more effective electron capture, which is expected to scale with the square of charge state [2] (the efficiency for ubiquitin is slightly lower than for melittin, but we believe that the ubiquitin efficiency is underestimated because of numerous low abundance fragment ions unobserved near the spectral baseline). Moreover, the optimal irradiation period scales inversely with charge state $(30 \mathrm{~ms}$ for glu-fibrinopeptide, $15 \mathrm{~ms}$ for melittin, and $5 \mathrm{~ms}$ for ubiquitin)-further evidence for a link between ion charge state and electron capture efficiency. Finally, it is obvious that there is further room for improvement (by a factor of 2 to 3, with even more improvement possible for doubly charged ions).

\section{Reflected Electrons Versus Single Pass Electrons}

We investigated two distinct methods for electron injection, a single pass of electrons through the ion cloud (in which unreacted electrons are collected after one

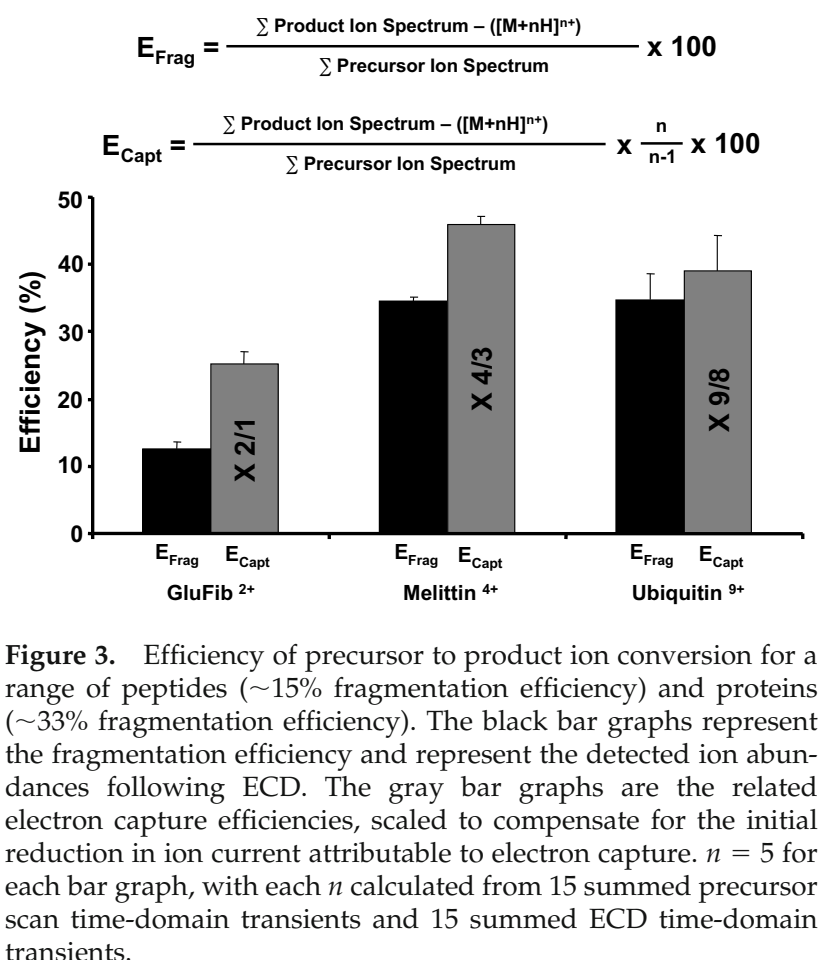




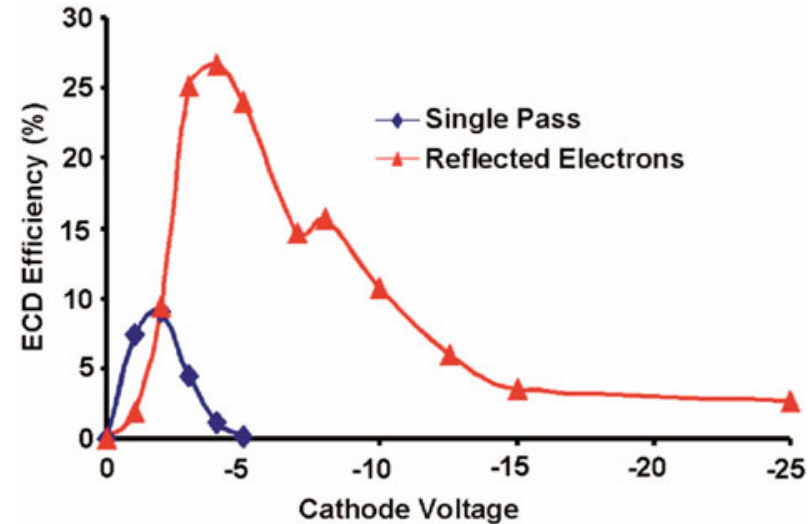

Figure 4. ECD fragmentation efficiency for a $30 \mathrm{~ms}$ irradiation event as a function of dispenser cathode voltage for a single pass of electrons (filled diamond) and electron beam reflection (filled triangle). Efficiency is much greater with reflected electrons and is less sensitive to cathode voltage.

pass through the system) and multiple reflections of the electrons through the ICR cell (Figure 1). In the single pass experiment, the DC offset on the transfer octopole is positively biased such that the electrons traversing the cell are deposited on the octopole rods. In the reflection experiment, electrons are trapped between the negatively biased transfer octopole and the cathode so that they make many passes through the ICR cell.

The two methods are compared in Figure 4 under optimal conditions for each for ECD of Substance P. The single pass method is strikingly less efficient and is effective over a much narrower electron energy range centered near $1 \mathrm{eV}$, in agreement with early ECD literature [1]. Further, we found that the optimal cathode bias drifts throughout the day, presumably due to change in the dispenser cathode surface temperature, which changes the electron energy. The reflection method is considerably more efficient and forgiving (Figure 4). In fact, we were able to achieve $\sim 25 \%$ efficiency, which is almost three times higher than for the single pass experiment and begins to approach the theoretical maximum (which is actually less than 50\% when fragment neutralization is considered [31]. Tsybin et al. have recently reported successful ECD over a similarly wide range of energy [32], and communication with the authors confirmed that electrons were refelcted. Further, we suspect that significant electron cooling takes place before electron capture (see below).

\section{Trapped Electrons}

Further evidence suggests that some of the reflected electrons are trapped in potential wells (for negatively charged species) near the ICR cell. We note that the electron reflection method requires a brief event after electron irradiation meant to remove electrons from the ICR cell region, as illustrated in Figure 5. Electron removal can be performed in two ways. Initially, we lowered the trapping electrode potentials (from 10 to 2
V) and raised the cathode and grid potentials for $1 \mathrm{~ms}$ to act as an electron dump. We later discovered that the trap potentials could be pulsed to more negative potentials $(0$ to $-10 \mathrm{~V}$, similar to a suspended trapping event [30] for a shorter period $(\sim 50 \mu \mathrm{s})$. The data in Figure 4 were collected with a $50 \mu$ s pulse to $-10 \mathrm{~V}$. The striking effect of trap voltage manipulation also supports the hypothesis that the electrons must be trapped in the (negative) potential wells at the center of each trapping electrode (see Figure 1). Potential reversal rapidly expels electrons but is completed so quickly that (much larger) ions don't have time to respond to the change in potential.

We have also found that the electron irradiation period can be significantly shortened if it is followed by a suitable delay. For example, equivalent ECD spectra of substance $\mathrm{P}$ are generated with a $500 \mu$ s electron irradiation period followed by a 29.5 ms delay (during which injected electrons can interact with the ion cloud but additional electrons are not allowed to enter the cell region), or a $30 \mathrm{~ms}$ irradiation period followed immediately by electron removal. Apparently, the trapped electrons cool over a period of milliseconds to an energy low enough for capture. The seminal description of ECD proposed an extra set of electrodes for trapping electrons [1,2]. Our results indicate that we can effectively trap electrons within an unmodified open-ended ICR cell by raising the trapping electrode potentials to $+10 \mathrm{~V}$ and biasing the transfer octopole at $-60 \mathrm{~V}$ during the ECD event. Electron trapping provides time for cooling, and therefore a wider range of cathode voltages produces electrons that promptly reach kinetic energy sufficient for capture. Consequently, the trapped electron method is much less sensitive to cathode voltage. Electron cooling can also be achieved by use of a collision gas in the ICR cell before interaction with

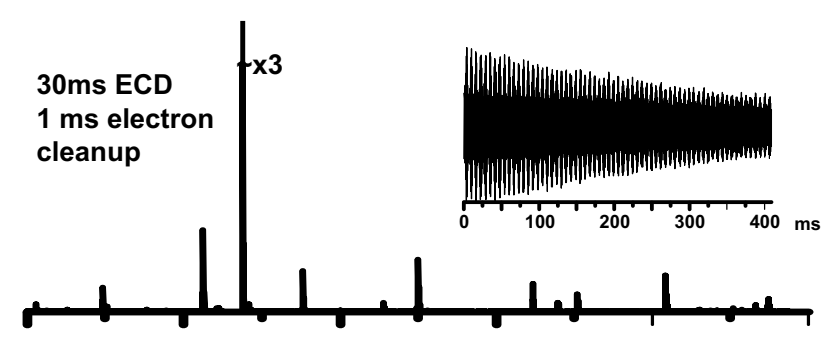

$30 \mathrm{~ms}$ ECD

no electron cleanup

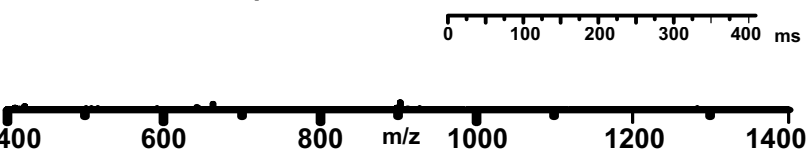

Figure 5. ECD FT-ICR mass spectra with (top) and without (bottom) removal of trapped electrons prior to ion excitation and detection. The corresponding time-domain transients are shown as insets. The y-axis scale is the same for both top and bottom spectra. Optimal performance requires electron removal. 
thermalized ions (plasma ECD) [33]. However, plasma ECD requires a substantial (30 s) pumpdown delay to remove the cooling gas. Future work will explore the nature of electron cooling, but it appears that electrons are trapped in the vicinity of the ICR cell and it is possible that they cool to an energy suitable for capture.

\section{Depletion of Precursor}

An especially troubling observation is that continued electron irradiation of the precursor ion does not result in its continued depletion. Rather, once the optimal irradiation period has passed, the abundances of all ions (precursors and products) decrease (Figure 2). Charge neutralization can account for some decrease in product ions, but cannot account for the plateau in parent ion signal. One explanation is that the electric field produced by the space charge of the electron beam is substantial enough to trap a population of ions within its own potential well [34]. Rather than rotating into the path of dissociation as expected for photodissociation, the remaining precursor ions rotate around the electron beam (because it has established a new electric field saddle point) and never come into contact with the electrons. Electrons then interact only with ions initially trapped in the electron trap and essentially "bleach" a hole through the ion cloud. We tested that hypothesis by performing sequential ECD events (Figure 6). Doubly protonated substance $P$ precursor ions were irradiated for $30 \mathrm{~ms}$ under trapped electron conditions. The subsequent product ions were ejected by SWIFT isolation and the remaining precursor ions irradiated again for $30 \mathrm{~ms}$. Few additional product ions were observed. Experiments were repeated with different (static) cathode voltages for the second ECD event, and by sweep of

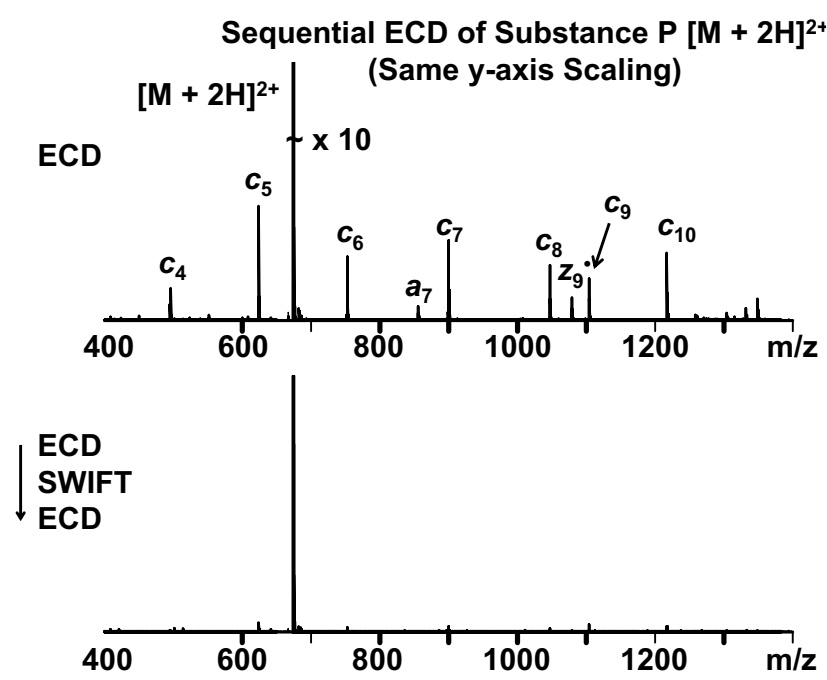

Figure 6. ECD FT-ICR mass spectra detected following a typical ECD experiment (top) and a sequence that includes ECD, SWIFT isolation of remaining precursor ions, and a second ECD event (bottom, see text). Strikingly few additional product ions are observed following the second ECD event. the cathode voltage during irradiation (data not shown). Neither set of experiments resulted in significant fragmentation, suggesting that the limitation is not a mismatch of ion and electron energies. Another possibility is that the ion cloud and electron beam are either misaligned radially or that the electron beam is much smaller diameter (or both) such that the remaining precursor ions are unable to move into the bleached area.

\section{Adjustable Probe Mounted Dispenser Cathode}

We tested the alignment and diameter of the electron beam by using a probe-mounted dispenser cathode. Normally, the cathode is $\sim 73 \mathrm{~cm}$ from the ICR cell center, just inside the magnet passive shield. The probemounted cathode could be moved axially from $\sim 28$ to $88 \mathrm{~cm}$ from the ICR cell center. The probe was also rotatable, such that the cathode could be placed radially on- and off-axis (by up to $2.5 \mathrm{~cm}$ ) with respect to the ICR cell axis. If a hole is bleached through the ion cloud, better alignment and/or a larger beam cross section should increase the ECD fragmentation efficiency.

The magnetic field at the dispenser cathode displaced $73 \mathrm{~cm}$ axially from magnet (and ICR cell) center is estimated (by the magnet manufacturer) to be $\sim 0.8$ tesla. Traversal of the magnetic field gradient from $0.8 \mathrm{~T}$ to $9.4 \mathrm{~T}$ at the cell center focuses the $1 \mathrm{~cm}$ electron beam by more than 3-fold [35] because the beam area scales inversely with field strength (and diameter by the square root of field strength). The magnetic field is estimated to be $\sim 8.6 \mathrm{~T}$ if the cathode is placed $\sim 28 \mathrm{~cm}$ from the ICR cell center, resulting in a 3 -fold wider beam diameter. We hoped that substantial increase in electron beam diameter would improve overlap between ions and electrons, and thus improve the observed ECD efficiency. However, no substantial change in the ECD spectra was observed. One possible explanation is that the overlap was already optimized and that no further improvement could be made. In fact, the initial electron beam diameter $(\sim 3 \mathrm{~mm})$ already exceeds that estimated for the ion cloud $(\sim 2 \mathrm{~mm})$. If misalignment between ions and electrons is negligible, no improvement would be expected. Consequently, fewer ions are trapped by the electron beam. Finally, changes in instrument configuration require a temporary instrument vent to atmospheric pressure. The surface of a dispenser cathode does not reach optimal performance until it has been operated over a period of time under vacuum (for conditioning and equilibration of the emitting surface). Possibly, more extended use would have resulted in improvement as the emitting surface was conditioned.

The adjustable probe did allow us to evaluate the ICR cell alignment. Several recent papers have suggested that ECD efficiency can be improved by spatial manipulation of the ion cloud [31,36]. We used dipolar and quadrupolar excitation $[37,38]$ to confirm that our ICR cell is nominally centered in the magnetic field. For 
example, we used dipolar excitation to move ions into the path of the off-axis cathode and induce ECD. Conversely, we were able to increase the radius of the ion cloud with the cathode on-axis and eliminate ECD. We were unable to improve upon our best ECD results by use of any ion manipulation. However, even a small ( $\sim 1 \mathrm{~mm})$ misalignment could have a significant impact on efficiency, and we shall report separately on this issue.

\section{Conclusions}

ECD with reflected (and ultimately trapped) electrons is more efficient and robust than a single pass of electrons, and can be achieved in an open ICR cell by appropriate bias of the ICR trap plates and ion injection optics during electron irradiation. An electron irradiation period of 5-30 ms is sufficient for a wide range of peptides and small proteins, with little tuning of potentials or electron beam current. Further, ECD with trapped electrons requires only a short (500 $\mu$ s) electron irradiation period followed by an appropriate delay for interaction. Electron removal (by, e.g., suspended trapping) following ECD is necessary for best efficiency and reproducibility. ECD efficiency of $15-33 \%$ (depending on ion charge state) is typical utilizing these techniques. However, 2- to 3-fold improvement is possible, but has not (as yet) been effected by manipulation of the physical alignment of the ion-electron interaction, the interaction period, and/or the relative energies.

\section{Acknowledgments}

The authors thank Steven C. Beu, Yury O. Tsybin, and Matthew B. Renfrow for helpful discussions. This work was supported by the NSF National High-Field FT-ICR Mass Spectrometry Facility (CHE 99-09,502), Florida State University, and the National High Magnetic Field Laboratory at Tallahassee, Florida.

\section{References}

1. Zubarev, R. A.; Kelleher, N. L.; McLafferty, F. W. Electron Capture Dissociation of Multiply Charged Protein Cations. A Nonergodic Processes J. Am. Chem. Soc. 1998, 120, 3265-3266.

2. Zubarev, R. A.; Horn, D. M.; Fridriksson, E. K.; Kelleher, N. L.; Kruger, N. A.; Lewis, M. A.; Carpenter, B. K.; McLafferty, F. W. Electron Capture Dissociation for Structural Characterization of Multiply Charged Protein Cations. Anal. Chem. 2000, 72, 563-573.

3. Håkansson, K.; Cooper, H. J.; Emmett, M. R.; Costello, C. E.; Marshall, A. G.; Nilsson, C. L. Electron Capture Dissociation and Infrared Multiphoton Dissociation MS/MS of an NGlycosylated Tryptic Peptide Yield Complementary Sequence Information. Anal. Chem. 2001, 73, 4530-4536.

4. Mirgorodskaya, E.; Roepstorff, P.; Zubarev, R. A. Localization of O-Glycosylation Sites in Peptides by Electron Capture Dissociation in a Fourier Transform Mass Spectrometer. Anal. Chem. 1999, 71, 4431-4436.

5. Stensballe, A.; Jensen, O. N.; Olsen, J. V.; Haselmann, K. F.; Zubarev, R. A. Electron Capture Dissociation of Singly and Multiply Phosphorylated Peptides. Rapid Commun. Mass Spectrom. 2000, 14, 1793-1800.
6. Kelleher, R. L.; Zubarev, R. A.; Bush, K.; Furie, B.; Furie, B. C.; McLafferty, F. W.; Walsh, C. T. Localization of Labile Posttranslational Modifications by Electron Capture Dissociation: The Case of $\gamma$-Carboxyglutamic Acid. Anal. Chem. 1999, 71, 4250-4253.

7. Chalmers, M. J.; Kolch, W.; Emmett, M. R.; Marshall, A. G.; Mischak, H. Identification and Analysis of Phosphopeptides. J. Chromatogr. B 2004, 803, 111-120.

8. Chalmers, M. J.; Håkansson, K.; Johnson, R.; Smith, R.; Shen, J.; Emmett, M. R.; Marshall, A. G. Protein Kinase A Phosphorylation Characterized by Tandem Fourier Transform Ion Cyclotron Resonance Mass Spectrometry. Proteomics 2004, 4, 970981.

9. Guan, Z. Identification and Localization of the Fatty Acid Modification in Ghrelin by Electron Capture Dissociation. J. Am. Soc. Mass Spectrom. 2002, 13, 1443-1447.

10. Fagerquist, C. K.; Hudgins, R. R.; Emmett, M. R.; Håkansson, K.; Marshall, A. G. An Antibiotic Linked to Peptides and Proteins is Released by Electron Capture Dissociation Fourier Transform Ion Cyclotron Resonance Mass Spectrometry. J. Am. Soc. Mass Spectrom. 2003, 14, 302-310.

11. Cooper, H. J.; Heath, J. K.; Jaffray, E.; Hay, R.; Lam, T.; Marshall, A. Identification of Sites of Ubiquitination in Proteins: A Fourier Transform Ion Cyclotron Resonance Mass Spectrometry Approach. Anal. Chem. 2004, 76, 6982-6988.

12. Schultz, K.; Håkansson, K. Rapid Electron Capture Dissociation of Mass-Selectively Accumulated Oligodeoxynucleotide Dications. Int. J. Mass Spectrom. 2004, 234, 123-130.

13. Zhang, L.; Freitas, M. Comparison of Peptide Mass Mapping and Electron Capture Dissociation as Assays for Histone Posttranslational Modifications. Int. J. Mass Spectrom. 2004, 234, 213-225.

14. Pesavento, J.; Kim, Y.; Taylor, G.; Kelleher, N. Shotgun Annotation of Histone Modifications: A New Approach for Streamlined Characterization of Proteins by Top Down Mass Spectrometry. J. Am. Chem. Soc. 2004, 126, 3386-3387.

15. Medzihradszky, K.; Zhang, X.; Chalkley, R.; Guan, S.; McFarland, M.; Chalmers, M.; Marshall, A.; Diaz, R.; Allis, C.; Burlingame, A. Characterization of Tetrahymena Histone H2B Variants and Posttranslational Populations by Electron Capture Dissociation (ECD) Fourier Transform Ion Cyclotron Mass Spectrometry (FTICRMS). Mol. Cell. Proteom. 2004, 3, 872-886.

16. Tsybin, Y. O.; Håkansson, P.; Budnik, B. A.; Haselmann, K. F.; Kjeldsen, F.; Gorshkov, M.; Zubarev, R. A. Improved LowEnergy Electron Injection Systems for High Rate Electron Capture Dissociation in Fourier Transform Ion Cyclotron Resonance Mass Spectrometry. Rapid Commun. Mass Spectrom. 2001, 15, 1849-1854.

17. Håkansson, K.; Chalmers, M. J.; Quinn, J. P.; McFarland, M. A.; Hendrickson, C. L.; Marshall, A. G. Combined Electron Capture and Infrared Multiphoton Dissociation for Multistage MS/MS in a Fourier Transform Ion Cyclotron Resonance Mass Spectrometer. Anal. Chem. 2003, 75, 3256-3262.

18. Senko, M. W.; Hendrickson, C. L.; PasaTolic, L.; Marto, J. A.; White, F. M.; Guan, S. H.; Marshall, A. G. Electrospray Ionization Fourier Transform Ion Cyclotron Resonance at 9.4 T. Rapid Commun. Mass Spectrom. 1996, 10, 1824-1828.

19. Quinn, J. P.; Emmett, M. R.; Marshall, A. G. A Device for Fabrication of Emitters for Low-Flow Electrospray Ionization. Proceedings of the 46th ASMS Conference on Mass Spectrometry and Allied Topics; Orlando, FL, May, 1998.

20. Senko, M. W.; Hendrickson, C. L.; Emmett, M. R.; Shi, S. D.-H.; Marshall, A. G. External Accumulation of Ions for Enhanced Electrospray Ionization Fourier Transform Ion Cyclotron Resonance Mass Spectrometry. J. Am. Soc. Mass Spectrom. 1997, 8, 970-976. 
21. Hendrickson, C. L.; Quinn, J. P.; Emmett, M. R.; Marshall, A. G. Quadrupole Mass Filtered External Accumulation for Fourier Transform Ion Cyclotron Resonance Mass Spectrometry. Proceedings of the 48th ASMS Conference on Mass Spectrometry and Allied Topics; Long Beach, CA, June, 2000.

22. Hendrickson, C. L.; Quinn, J. P.; Emmett, M. R.; Marshall, A. G. Mass-Selective External Ion Accumulation for Fourier Transform Ion Cyclotron Resonance Mass Spectrometry. Proceedings of the 49th ASMS Conference on Mass Spectrometry and Allied Topics; Chicago, IL, May, 2001.

23. Wilcox, B. E.; Hendrickson, C. L.; Marshall, A. G. Improved Ion Extraction from a Linear Octopole Ion Trap: SIMION Analyis and Experimental Demonstration. J. Am. Soc. Mass Spectrom. 2002, 13, 1304-1312.

24. Beu, S. C.; Laude, D. A., Jr. Open Trapped Ion Cell Geometries for FT/ICR/MS. Int. J. Mass Spectrom. Ion Processes 1992, 112, 215-230.

25. Marshall, A. G.; Wang, T.-C. L.; Ricca, T. L. Tailored Excitation for Fourier Transform Ion Cyclotron Resonance Mass Spectrometry. J. Am. Chem. Soc. 1985, 107, 7893-7897.

26. Guan, S.; Marshall, A. G. Stored Waveform Inverse Fourier Transform (SWIFT) Ion Excitation in Trapped-Ion Mass Spectrometry: Theory and Applications. Int. J. Mass Spectrom. Ion Processes 1996, 157/158, 5-37.

27. Ledford, E. B., Jr.; Rempel, D. L.; Gross, M. L. Space Charge Effects in Fourier Transform Mass Spectrometry Mass Calibration. Anal. Chem. 1984, 56, 2744-2748.

28. Shi, S. D.-H.; Drader, J. J.; Freitas, M. A.; Hendrickson, C. L.; Marshall, A. G. Comparison and Interconversion of the Two Most Common Frequency-to-Mass Calibration Functions for Fourier Transform Ion Cyclotron Resonance Mass Spectrometry. Int. J. Mass Spectrom. 2000, 195/196, 591-598.

29. Senko, M. W.; Canterbury, J. D.; Guan, S.; Marshall, A. G. A High-Performance Modular Data System for FT-ICR Mass Spectrometry. Rapid Commun. Mass Spectrom. 1996, 10, 1839-1844.
30. Laude, D. A., Jr.; Beu, S. C. Suspended Trapping Pulse Sequence for Simplified Mass Calibration in FTMS. Anal. Chem. 1989, 61, 2422-2427.

31. Gorshkov, M. V.; Masselon, C. D.; Nikoleav, E. N.; Udseth, H. R.; PasaTolic, L.; Smith, R. D. Considerations for Electron Capture Dissociation Efficiency in FT-ICR Mass Spectrometry. Int. J. Mass Spectrom. 2004, 234, 131-136.

32. Tsybin, Y. O.; Witt, M.; Baykut, G.; Håkansson, P. Electron Capture Dissociation Fourier Transform Ion Cyclotron Resonance Mass Spectrometry in the Electron Energy Range 0-50 eV. Rapid Commun. Mass Spectrom. 2004, 18, 1607-1613.

33. Sze, S. K.; Ge, Y.; Oh, H. B.; McLafferty, F. W. Plasma Electron Capture Dissociation for the Characterization of Large Proteins by Top Down Mass Spectrometry. Anal. Chem. 2003, 75, 1599-1603.

34. Hendrickson, C. L.; Hadjarab, F.; Laude, D. A. J. Electron Beam Potential Depression as an Ion Trap in Fourier Transform Ion Cyclotron Resonance Mass Spectrometry. Int. J. Mass Spectrom. Ion Processes 1995, 141, 161-170.

35. Alford, J. M.; Williams, P. E.; Trevor, D. J.; Smalley, R. E. Metal Cluster ICR. Combining Supersonic Metal Cluster Beam Technology with FT-ICR. Int. J. Mass Spectrom. Ion Processes 1986, $72,33-51$

36. Mormann, M.; Peter-Katalinic, J. Improvement of Electron Capture Dissociation Efficiency by Resonant Excitation. Rapid Commun. Mass Spectrom. 2003, 17, 2208-2214.

37. Hendrickson, C. L.; Drader, J. J.; Laude, D. A., Jr. Simplified Application of Quadrupolar Excitation in Fourier Transform Ion Cyclotron Resonance Mass Spectrometry. J. Am. Soc. Mass Spectrom. 1995, 6, 448-452.

38. Jackson, G. S.; Hendrickson, C. L.; Reinhold, B. B.; Marshall, A. G. Two-Plate versus Four-Plate Azimuthal Quadrupolar Excitation for FT-ICR Mass Spectrometry. Int. J. Mass Spectrom. Ion Processes 1997, 165/166, 327-338. 